\title{
3-D Ultrasound Imaging Performance of a Row-Column Addressed 2-D Array Transducer: A Measurement Study
}

\section{Rasmussen, Morten Fischer; Jensen, Jørgen Arendt}

Published in:

2013 IEEE International Ultrasonics Symposium Proceedings.

Link to article, DOI:

10.1109/ULTSYM.2013.0370

Publication date:

2013

Document Version

Early version, also known as pre-print

Link back to DTU Orbit

Citation (APA):

Rasmussen, M. F., \& Jensen, J. A. (2013). 3-D Ultrasound Imaging Performance of a Row-Column Addressed 2-D Array Transducer: A Measurement Study. In 2013 IEEE International Ultrasonics Symposium Proceedings. (pp. 1460-1463). IEEE. https://doi.org/10.1109/ULTSYM.2013.0370

\section{General rights}

Copyright and moral rights for the publications made accessible in the public portal are retained by the authors and/or other copyright owners and it is a condition of accessing publications that users recognise and abide by the legal requirements associated with these rights.

- Users may download and print one copy of any publication from the public portal for the purpose of private study or research.

- You may not further distribute the material or use it for any profit-making activity or commercial gain

- You may freely distribute the URL identifying the publication in the public portal 
Paper presented at the IEEE Internation Ultrasonics Symposium 2013:

\section{3-D Ultrasound Imaging Performance of a Row-Column Addressed 2-D Array Transducer: A Measurement Study}

Morten Fischer Rasmussen and Jørgen Arendt Jensen

Center for Fast Ultrasound Imaging,

Biomedical Engineering Group,

Department of Electrical Engineering

Ørsteds Plads Building 349,

Technical University of Denmark,

2800 Kgs. Lyngby, Denmark. 


\title{
3-D Ultrasound Imaging Performance of a Row-Column Addressed 2-D Array Transducer: A Measurement Study
}

\author{
Morten Fischer Rasmussen and Jørgen Arendt Jensen \\ Center for Fast Ultrasound Imaging, Department of Electrical Engineering, Technical University of Denmark, \\ 2800 Kgs. Lyngby, Denmark
}

\begin{abstract}
A real-time 3-D ultrasound measurement using only 32 elements and 32 emissions is presented. The imaging quality is compared to a conventionally fully addressed array using $\mathbf{1 0 2 4}$ elements and 256 emissions. The main-lobe of the measured line spread function is almost identical, but the side-lobe levels are higher for the row-column addressed array. The cystic resolution sampled at a relative intensity difference of $20 \mathrm{~dB}$ shows a cyst size of $5.00 \mathrm{~mm}$ for the row-column addressed array and $2.39 \mathrm{~mm}$ for the fully sampled array. A simulation study is carried out which compares how the imaging quality of the two addressing methods scales with the number of beamforming channels used. It is shown that for any fixed number of active elements, a rowcolumn addressed array achieves a better image quality than fully addressing the array. When using 128 channels, the mainlobe when fully addressing the array is $\mathbf{5 1 0} \%$ larger than when row-column addressing the array. The cyst radius needed to achieve $-20 \mathrm{~dB}$ intensity in the cyst is $\mathbf{3 9 6} \%$ larger for the fully addressed array compared to the row-column addressed array. The measurements were made using the experimental ultrasound scanner SARUS and a 32x32 element ultrasound probe made by Vermon S.A.
\end{abstract}

\section{INTRODUCTION}

For ultrasonic 3-D imaging, 2-D array transducers are required to achieve real-time scanning of a volume [1], [2]. The number of elements in a fully addressed 2-D array scales with the square of the number of elements in each dimension. To control all elements in the array, a connection to each element is necessary. However, addressing each element individually results in a vast amount of interconnections and a large amount of data to be recorded. This posses both a great practical challenge in producing the internections and in sampling and real-time processing the large amount of data. An $N \times N$ element array can be operated using just $2 N$ connections when row-column addressing is used [3]-[7]. This is opposed to the $N^{2}$ connections needed when conventionally addressing the elements. An earlier publication by the authors investigated the 3-D imaging performance of row-column addressed arrays via simulations [7]. The simulations indicated that a good imaging performance is achievable when apodizing the sub-elements within each row and column element, thereby suppressing the otherwise distinct ghost echoes. In this paper the imaging performance of a $32+32$ element row-column addressed array is investigated. The investigation is made through measurements carried out using the 1024 channel research ultrasound scanner SARUS and a $32 \times 32$ element

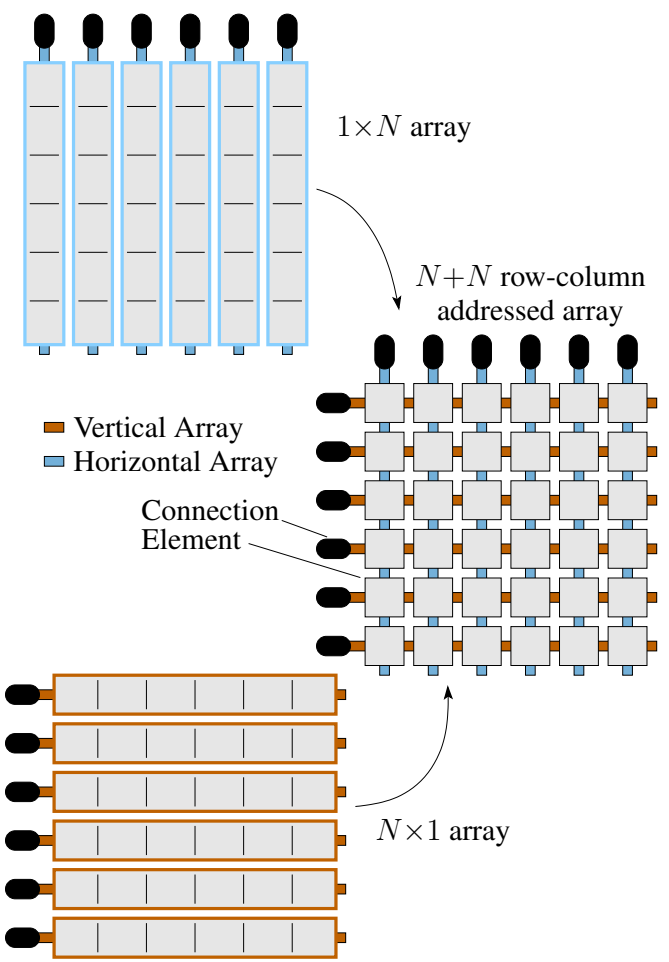

Figure 1. A row-column addressed 2-D array can be interpreted as two orthogonal 1-D arrays.

ultrasound probe. The measurements are compared to a fully addressed array using the 'gold standard' for real-time 3-D ultrasound imaging: Explososcan. It is also investigated through Field II simulations how the imaging performance scales with the number of active elements used for both the fully addressed array and the row-column addressed array.

\section{Methods}

\section{A. Row-column Addressed Array}

The principle of row-column addressing a 2-D array is to address the elements by their row- or column index, thus addressing an entire row or column of elements instead of a single element. This effectively turns the 2-D array into two orthogonal 1-D arrays, which is illustrated in Fig. 1. The first 1-D array consists of row-elements and the second 1-D array of column elements. Each element in the 2-D array is now a 
line element, either a row element or a column element, and each line element consists of $N$ sub-elements.

As is shown in [5] and [7] the creation of large row and column elements leads to edge effects. The more sub-elements a line element consists of, the more apparent the edge effects becomes. To decrease the edge effects, the sub-elements are apodized with a window function that tends to zero towards the edges of the line elements. In this paper the sub-elements are apodized with a Hann-window.

For the measurements a 2-D row-column addressed array with $32+32$ elements is emulated using a $32 \times 32$ fully addressed array. For the Field II simulations a $N+N$ element array is emulated by using a $N \times N$ element array. In transmit all elements in the same row are used as one element; they emit the same signal with the same time delay. The focus zone therefore has the shape of a line segment. Synthetic transmit focusing (STF) is used to focus the image in the elevation direction ( $y$-dimension). In receive, the signals measured from elements in the same column are summed to one signal before being dynamically focused in the lateral dimension ( $x$-dimension). The elevation direction can thereby be focused in transmit and the lateral direction in receive. Because of limitations in the beamforming software the emissions are focused using a single line element at a time. The entire 3-D volume is therefore acquired using $N$ emissions for an $N+N$ element array.

The emitted wavefront of a single element has the shape of a cylinder surface: it is a plane wave in the $z-x$ plane and a circle arc in $z-y$ plane. This is illustrated in Fig. 2. The time of flight is given by the shortest distance from the source to the scatterer and back to the receiving element, divided by the speed of sound. Using the notation from Fig. 2, this can be written as:

$$
t_{T o F}=\frac{\left\|\mathbf{r}_{\mathbf{e}}\right\|+\left\|\mathbf{r}_{\mathbf{r}}\right\|}{c},
$$

where $r_{e}$ is the vector from the emitting element to the focus point, $\mathbf{r}_{\mathbf{r}}$ is the vector from the focus point and back to the receiving element, and $c$ is the speed of sound in the medium. Since the length of each element is large, the usual assumption that the elements are point sources no longer holds. Therefore, $\mathbf{r}_{\mathbf{e}}$ and $\mathbf{r}_{\mathbf{r}}$ connects the focus point to the closest point on the source element and the receiving element respectively.

Determining $\left\|\mathbf{r}_{\mathbf{e}}\right\|$ and $\left\|\mathbf{r}_{\mathbf{r}}\right\|$ is solved by calculating the distance between an arbitrary point in $3-\mathrm{D}$, the focus point $\mathbf{P}$, and a line segment $\mathbf{A B}$ from point $\mathbf{A}$ to point $\mathbf{B}$. The minimum distance between the point $\mathbf{P}$ and the line segment $\mathbf{A B}$ is in [7] shown to be:

$$
d(\mathbf{A B}, \mathbf{P})= \begin{cases}\frac{\|\mathbf{A B} \times \mathbf{A P}\|}{\|\mathbf{A B}\|} & \text { if } 0 \leq \hat{s} \leq 1, \\ \|\mathbf{A P}\| & \text { if } \hat{s}<0, \\ \|\mathbf{B P}\| & \text { if } \hat{s}>1,\end{cases}
$$

where $\hat{s}$ is determined by:

$$
\hat{s}=\frac{\mathbf{A P} \cdot \mathbf{A B}}{\|\mathbf{A B}\|^{2}} .
$$

Transmit Array

Receive Array

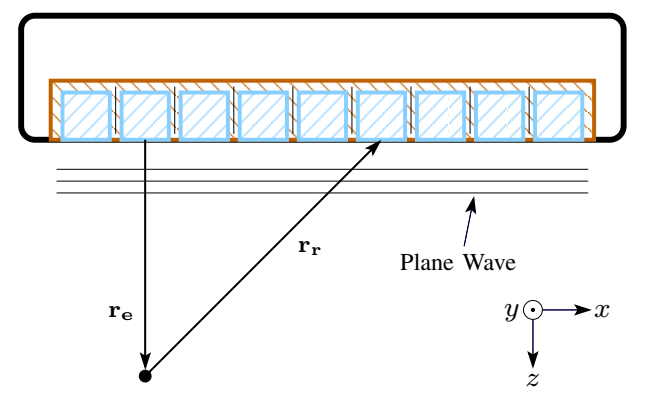

(a) ToF in the $x$-dimension.

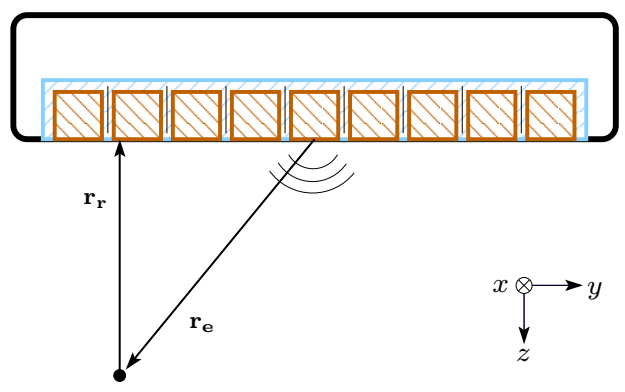

(b) ToF in the $y$-dimension.

Figure 2. Time of flight (ToF) illustration of one emission to the focus point and back to one receiving element. $r_{e}$ is the vector from the nearest point of the emitting element to the focus point and $\mathbf{r}_{\mathbf{r}}$ is the vector from the focus point to the nearest point on the receive element.

Using (2), the distances $\left\|\mathbf{r}_{\mathbf{e}}\right\|$ and $\left\|\mathbf{r}_{\mathbf{r}}\right\|$ can now be determined as

$$
\left\|\mathbf{r}_{\mathbf{e}}\right\|=d\left(\mathbf{x m t}_{m}, \mathbf{P}\right) \text { and }\left\|\mathbf{r}_{\mathbf{r}}\right\|=d\left(\mathbf{r c v}_{n}, \mathbf{P}\right),
$$

where $\mathbf{x m t}_{m}$ is the $\mathbf{A}$ and $\mathbf{B}$ coordinates of the $m$ 'th transmit element and $\mathbf{r c v}_{n}$ is the corresponding coordinates for the $n$ 'th receive element.

\section{B. Fully Addressed Array}

Explososcan is used as the imaging technique for the fully addressed array. Explososcan is a parallel processing technique developed by Smith, von Ramm and colleagues in the 1980s [1], [8] which increases the acquisition rate compared to standard sequential acquisition. A broad transmit beam is emitted and multiple receive beams are focused in parallel during receive. If for instance $5 \times 5$ lines are beamformed per emission instead of just one, the frame rate increases by a factor of 25 . This paper beamforms $4 \times 4$ lines in receive for each emission. The focus point is placed at a depth of $60 \mathrm{~mm}$ and the $N$ closest elements to the center of the aperture are used in both transmission and in receive. The active aperture therefore has the shape of a circle, except when using 1024 active elements which corresponds to all elements in the $32 \times 32$ element array. 16 emissions are used per dimension, giving 256 emissions per imaged volume.

\section{Image Quality}

The imaging quality is investigated by acquiring the point spread function (PSF) of the simulations and the line spread function (LSF) of the measurements. The LSF is measured 
Table I

ULTRASOUND PROBE PARAMETERS.

\begin{tabular}{lcc}
\hline \hline Parameter name & Notation & Value \\
\hline Emission center frequency & $f_{0}$ & $2.6 \mathrm{MHz}$ \\
Transducer fundamental resonance freq. & $f_{T}$ & $3.5 \mathrm{MHz}$ \\
Number of transmitted cycles & $N_{\text {cycles }}$ & 2 \\
Probe pitch -x & $d_{x}$ & $300 \mu \mathrm{m}$ \\
Probe pitch -y & $d_{y}$ & $300 \mu \mathrm{m}$ \\
Total number of elements -x & $N_{\text {tot_x }}$ & 32 \\
Total number of elements $-\mathrm{y}$ & $N_{\text {tot_y }}$ & 35 \\
Inactive rows & - & $9,18,27$ \\
\hline \hline
\end{tabular}

on a wire phantom using both with a row-column addressed array and a fully addressed array. The 3-D volume is sliced across the direction of the wire, into a 2-D B-mode image, to emulate the point-spread-function (PSF).

The PSFs are evaluated using both the full-width-at-halfmaximum (FWHM) and the cystic resolution. The cystic resolution describes the ability to detect an anechoic cyst in a uniform scattering medium [9]. The lower the intensity is at the center of the cyst, i.e. the darker it appears, the better imaging performance. The relative intensity (RI) of the anechoic cyst was shown by Ranganathan and Walker [10], to be quantized as the clutter energy to total energy ratio,

$$
\mathrm{RI}(R)=\sqrt{\frac{E_{\text {out }}(R)}{E_{\text {tot }}}}=\sqrt{1-\frac{E_{\text {in }}(R)}{E_{\text {tot }}}},
$$

where $E_{\text {in }}$ is the signal energy inside a circular region with radius, $R$, centered on the peak of the point spread function, $E_{\text {tot }}$ is the total point spread function energy, and $E_{\text {out }}$ is the point spread function energy outside the circular region. The $\mathrm{RI}(R)$-curve can be compressed to a single number by sampling the curve at $20 \mathrm{~dB}$. The result is the required cyst radius at which the intensity at the cyst center is $20 \mathrm{~dB}$ lower than its surroundings, written as $R_{20 \mathrm{~dB}}$.

\section{Comparison}

As is seen from Table I the ultrasound probe contains three inactive rows of elements. This discontinuity in the aperture gives rise to higher side-lobes. When using few active elements in the aperture, fewer of the inactive rows become part of the active aperture. A smaller aperture therefore performs relatively better than a aperture using all available transducer elements. A direct comparison is therefore not feasible between for instance a $16+16$ and a $32 \times 32$ element array.

The image quality comparison for the measurements is made only between a $32+32$ row-column addressed array and a $32 \times 32$ element fully addressed array.

To compare how the image quality scales with the number of elements used, a dense aperture is simulated using Field II.

A Field II simulation study is carried out to compare how the image quality scales with the number of active element used. The row-column addressed array is simulated using 16, 32, 64 and 128 active elements and Explososcan is simulated using 32, 64, 128, 256, 512 and 1024 active elements. The simulated apertures have the same element size, pitch and

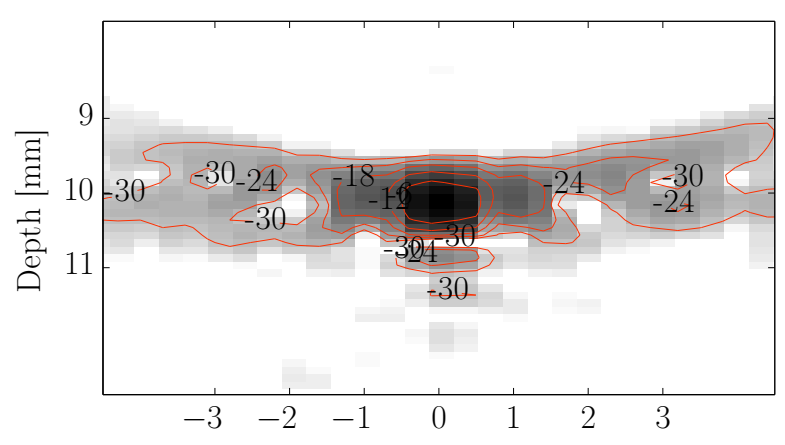

(a)

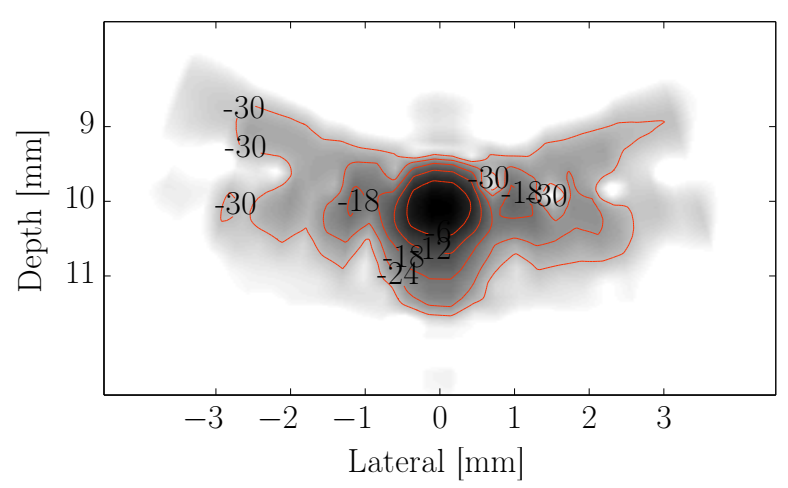

(b)

Figure 3. Line Spread Function of (a) Row-column addressed array 32 channels and 32 emissions and (b) Conventional fully addressed array with 1024 channels and 256 emissions.

frequency response as the $32 \times 32$ element ultrasound probe used for the measurements. The difference between the real and the simulated array being that the simulated array contains no discontinuities and an array size up to $128 \times 128$ element array is used.

\section{EXPERIMENTAL SETUP}

The measurements are performed using a 2-D phased array ultrasound probe, made by Vermon S.A., Tours, France, used. The 2 -D probe has $35 \times 32$ elements, of which row 9,18 and 27 are inactive, effectively giving $32 \times 32$ individually controllable elements. The ultrasound probe parameters are listed in Table I. The fundamental resonance frequency of the probe is $3.5 \mathrm{MHz}$, but in order for the element pitch to equal half a wavelength the center frequency of the emission is $2.6 \mathrm{MHz}$.

All measurements are carried out using the 1024 channel experimental ultrasound scanner SARUS (synthetic aperture real-time ultrasound system)[11]. The measured data are beamformed using the toolbox BFT3 [12].

When acquirering the LSF a $0.07 \mathrm{~mm}$ thick and $10 \mathrm{~cm}$ long cobber wire is placed in water $10 \mathrm{~mm}$ under the ultrasound probe surface. The wire is oriented parallel to the $y$-axis of the ultrasound probe. The wire is positioned using the positioner of the intensity measurement system "AIMS 3" made by Onda Corporation, Sunnyvale, CA, USA.

The simulation study of the image quality scaling is carried out using Field II [13], [14] and MATLAB (The MathWorks, 
Inc., Natick, Massachusetts, USA).

\section{RESULTS}

The measured LSF is seen in Fig. 3. The main-lobe size of the LSF is almost identical for the two measurements. The FWHM is $0.89 \mathrm{~mm}$ when row-column addressing the array and when fully addressing the array it is $0.86 \mathrm{~mm}$. A larger difference is apparent on the size of the side-lobes. When fully addressing the array a cystic resolution at $20 \mathrm{~dB}$ relative intensity difference, the $R_{20 \mathrm{~dB}}$, of $2.39 \mathrm{~mm}$ is achieved, whereas the $R_{20 \mathrm{~dB}}$ when row-column addressing the array is $5.00 \mathrm{~mm}$. The row-column addressed array thereby has a $3.4 \%$ larger main-lobe and a $109 \%$ larger $R_{20 \mathrm{~dB}}$. The better image quality of the fully addressed array comes with the cost of using 32 times as many elements and 8 times as many emissions than when row-column addressing the array.

In Fig. 4 the result of the simulation study is shown. For any given number of active channels row-column addressing an array is seen to give a better imaging quality than fully addressing the array. When using 128 channels the main-lobe is $510 \%$ larger fully addressing the array than when row-column addressing the array. The $R_{20 \mathrm{~dB}}$ increases $396 \%$ when fully addressing the array.

\section{CONCLUSion}

A wire phantom measurement made with an array that was both conventionally fully addressed and row-column addressed was presented. It was thereby demonstrated that full 3-D ultrasound imaging can be achieved using only 32 channels and 32 emissions. The measurement showed an almost identical main-lobe size, but larger side-lobes for the rowcolumn addressed array compared to the fully addressed array. The main-lobe was $3.4 \%$ larger and the $R_{20 \mathrm{~dB}} 109 \%$ larger for the row-column addressed array compared to the fully addressed array. This has to be kept in relation to that the fully addressed array used $3200 \%$ more elements and $800 \%$ as many emissions than when row-column addressing the array.

The simulation study showed that for any fixed number of active elements a row-column addressed array achieves better imaging quality than fully addressing the array.

\section{REFERENCES}

[1] O. T. von Ramm, S. W. Smith, and H. G. Pavy, "High-speed ultrasound volumetric imaging system - Part II: Parallel processing and image display," IEEE Trans. Ultrason., Ferroelec., Freq. Contr., vol. 38, pp. 109-115, 1991.

[2] D. Turnbull and F. Foster, "Beam steering with pulsed two-dimensional transducer arrays," IEEE Trans. Ultrason., Ferroelec., Freq. Contr., vol. 38, no. 4, pp. 320-333, July 1991.

[3] C. E. Morton and G. R. Lockwood, "Theoretical assessment of a crossed electrode 2-D array for 3-D imaging," in Proc. IEEE Ultrason. Symp., 2003, pp. 968-971.

[4] N. M. Daher and J. T. Yen, "Rectilinear 3-D ultrasound imaging using synthetic aperture techniques," in Proc. IEEE Ultrason. Symp., vol. 2, 2004, pp. 1270-1273.

[5] C. E. M. Démoré, A. Joyce, K. Wall, and G. Lockwood, "Real-time volume imaging using a crossed electrode array," IEEE Trans. Ultrason., Ferroelec., Freq. Contr., vol. 56, no. 6, pp. 1252-1261, 2009.

[6] C. H. Seo and J. T. Yen, "A 256 x 256 2-D array transducer with rowcolumn addressing for 3-D rectilinear imaging," IEEE Trans. Ultrason., Ferroelec., Freq. Contr., vol. 56, no. 4, pp. 837-847, apr 2009.

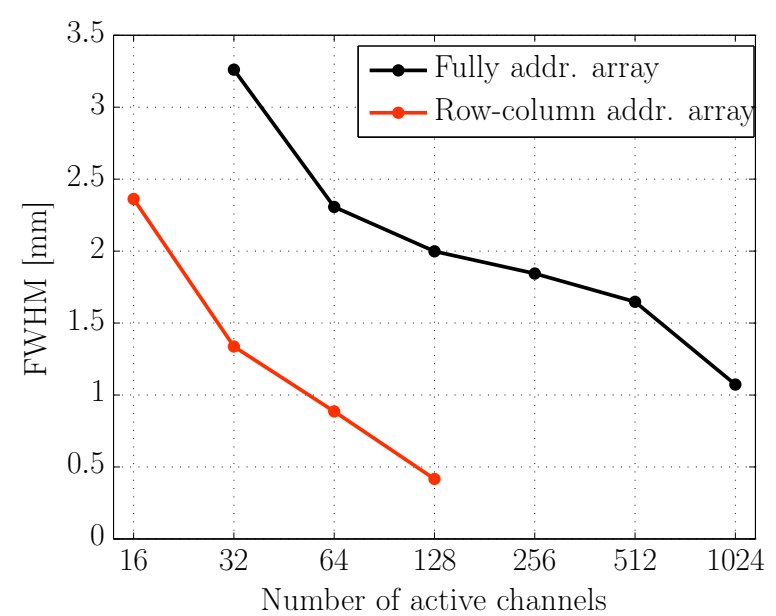

(a)

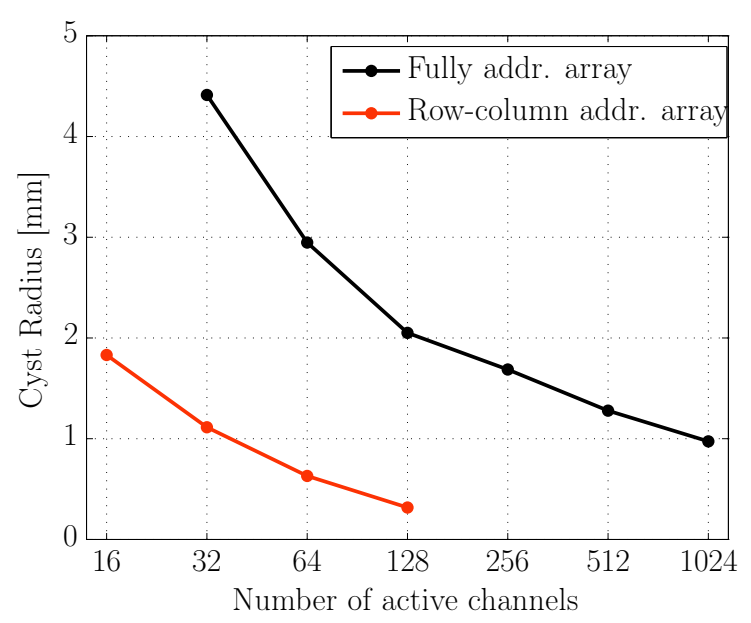

(b)

Figure 4. Resolution scaling with number og active channels.

[7] M. F. Rasmussen and J. A. Jensen, "3D ultrasound imaging performance of a row-column addressed 2D array transducer: a simulation study," in Proc. SPIE Med. Imag., 2013, pp. 1-11, 86750C.

[8] D. P. Shattuck, M. D. Weinshenker, S. W. Smith, and O. T. von Ramm, "Explososcan: A parallel processing technique for high speed ultrasound imaging with linear phased arrays," J. Acoust. Soc. Am., vol. 75, pp. 1273-1282, 1984.

[9] D. Vilkomerson, J. Greenleaf, and V. Dutt, "Towards a Resolution Metric for Medical Ultrasound Imaging," in Proc. IEEE Ultrason. Symp., 1995, pp. 1405-1410.

[10] K. Ranganathan and W. F. Walker, "Cystic Resolution: A Performance Metric for Ultrasound Imaging Systems," IEEE Trans. Ultrason., Ferroelec., Freq. Contr., vol. 54, no. 4, pp. 782-792, 2007.

[11] J. A. Jensen, H. Holten-Lund, R. T. Nilsson, M. Hansen, U. D. Larsen, R. P. Domsten, B. G. Tomov, M. B. Stuart, S. I. Nikolov, M. J. Pihl, Y. Du, J. H. Rasmussen, and M. F. Rasmussen, "SARUS: A synthetic aperture real-time ultrasound system," IEEE Trans. Ultrason., Ferroelec., Freq. Contr., p. In press, 2013.

[12] J. M. Hansen, M. C. Hemmsen, and J. A. Jensen, "An object-oriented multi-threaded software beamformation toolbox," in Proc. SPIE Med. Imag., vol. 7968, March 2011, pp. 79680 Y 1-9. [Online]. Available: http://dx.doi.org/10.1117/12.878178

[13] J. A. Jensen and N. B. Svendsen, "Calculation of Pressure Fields from Arbitrarily Shaped, Apodized, and Excited Ultrasound Transducers," IEEE Trans. Ultrason., Ferroelec., Freq. Contr., vol. 39, pp. 262-267, 1992.

[14] J. A. Jensen, "Field: A program for simulating ultrasound systems," Med. Biol. Eng. Comp., vol. 10th Nordic-Baltic Conference on Biomedical Imaging, Vol. 4, Supplement 1, Part 1, pp. 351-353, 1996. 